\title{
A Type of Directly Self-adaptive PID Controller Based on Neural Network
}

\author{
Han Chong \\ Commercial Aircraft Corporation of China, Jinke Road NO.5188, Shanghai, China \\ hanchong@comac.cc
}

Keywords: neural network; PID control; nonlinearity; learning algorithm

\begin{abstract}
A type of directly self-tuning PID controller based on neural network is proposed in this paper. Its main characteristic is that it no longer includes independent PID controller and put neural network and the law of PID controller together. Showing the learning algorithm of this neural network controller and analyzing the stability of this control system. The simulated results prove that this kind of control system is more adaptive and robust.
\end{abstract}

\section{Introduction}

PID arithmetic is in a dominant position in the industry control because of these advantages: simple architecture, steady operation and great robustness. But, as the development of industry, the object is more complex, especially for the time-varying and nonlinear complex system. And people's demand for the control quality is increasing day by day. The defects of common PID control exposed gradually.

In recent years, the rapid development of a new cross subject-artificial neural network has attracted great attention. The artificial neural network has strong ability of information integration, learning memory, self-adaption and approximating any nonlinear function. It can deal with process that can't be described by model and regulation. According to these unique characters, people put forward many intelligent PID control methods. Among these control methods, neural network is usually used auxiliary selection or self-adapted setting PID parameters, that can't overcome the contradiction between quickness and overshoot existed in linear combination. In this paper, a type of neural network self-adaptive PID controller is put forward. The relevant literature name it is "PID type neural network", "PID control with neural network structure". The character of this PID controller is that the proportion, integration and differentiation of error signals and self-adaptive setting of PID parameters are finished in a forward neural network. The experiment proved that this kind of controller has self-adaption and self-learning function, it has positive signification to improve the control effect and robustness of control system with strong nonlinear and rapid time varying characteristics.

\section{The Architecture of Self-adaptive PID Control System Based on Neural Network}

Neural network self-adaptive PID control system is as shown in figure 1 . This paper takes the $2 \times 3 \times 1$

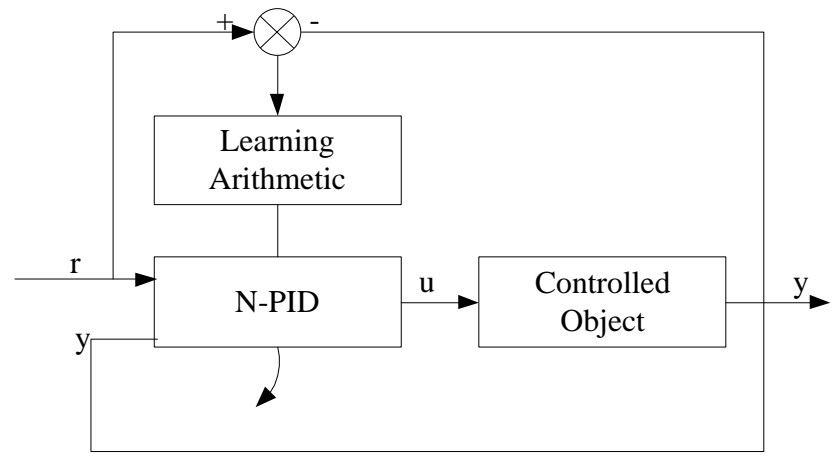

Fig. 1 Neural Network Self-Adaptive PID Control 
three layers network structure for example to explain how it works. The network N-PID structure is as shown in figure 2 . Two nodes of input layer are the given value $r(k)$ and controlled object value $y(k)$.

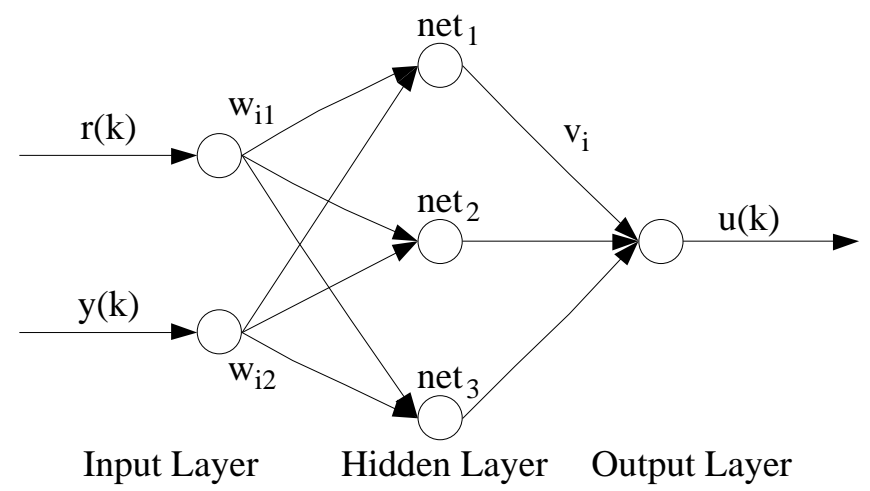

Fig. 2 Network N-PID Structure

Three nodes of hidden layer are used to do proportion, integration and differentiation operation of error signal. The output layer finishes integration of nonlinear PID control disciplinarian. The coefficients of P, I and D are reflected by network weight. The network weight value is adaptively adjusted according to choosing regulation through system error. The adaptive learning from input layer to hidden layer is used to filer the output $\mathrm{y}(\mathrm{k})$ disturbed random by measurement noise. The weight value adaptive adjusting from hidden layer to output layer is used to find the suitable PID parameters.

Setting $w_{i j}(k)(i=1,2,3 ; j=1,2)$ is the weight value from the first $\mathrm{j}$ unit of input layer to the first $\mathrm{i}$ unit of output layer. Assuming $\mathrm{j}=1$ of input layer unit corresponds to $r(\mathrm{k}), \mathrm{j}=2$ correspond to $\mathrm{y}(\mathrm{k}) ; v_{i}(\mathrm{i}=1$, $2,3)$ is the weight value from the first $i$ unit of hidden layer to output layer; net ${ }_{i}(i=1,2,3)$ is the total input of the first $i$ unit of hidden layer, $o_{i}(i=1,2,3)$ is the total output of the first $i$ unit of hidden layer.

The input of network hidden layer is:

$\operatorname{net}_{i}(k)=w_{i 1 r}(k)+w_{i 2} y(k),(i=1,2,3)$

The output of each node is:

$$
\begin{aligned}
& o_{1}(k)=f\left[\operatorname{net}_{1}(k)\right] \\
& o_{2}(k)=f\left[\operatorname{net}_{2}(k)\right]+f\left[\operatorname{net}_{2}(k-1)\right] \\
& o_{3}(k)=f\left[\operatorname{net}_{3}(k)\right]-f\left[\operatorname{net}_{3}(k-1)\right]
\end{aligned}
$$

Among them, $\mathrm{f}(\mathrm{x})$ is the output transform function and is set to $f(x)=\tanh (x)$. The output is set to this because that the proportion, integration and differentiation operation of error signal should be finished by it.

The network output is the control signal:

$u(k)=\sum_{i=1}^{3} v_{i} O_{i}(k),(i=1,2,3)$

\section{The Learning algorithm of Network Controller N-PID}

The performance function of choosing network is:

$$
J(k)=\frac{1}{2}[r(k+1)-y(k+1)]^{2}=\frac{1}{2} e^{2}(k+1)
$$

revising network weight value according to the steepest descent method, $v_{i}(i=1,2,3)$ and $w_{i j}(i=1,2,3 ; j=1,2)$ 
there is:

$$
\begin{aligned}
\Delta v(k)= & -\eta \frac{\partial J}{\partial v_{i}}=\eta e(k+1) \frac{\partial y(k+1)}{\partial u(k)} \cdot \frac{\partial u(k)}{\partial v_{i}} \\
& =\eta e(k+1) o_{i}(k) \cdot \frac{\partial y(k+1)}{\partial u(k)} \quad(i=1,2,3) \\
\Delta w_{i 1}(k) & =-\eta \frac{\partial J}{\partial w_{i 1}}=\eta e(k+1) \frac{\partial y(k+1)}{\partial u(k)} \cdot \frac{\partial u(k)}{\partial w_{i 1}}
\end{aligned} \quad \quad(i=1,2,3)
$$

Among them, $\eta$ is learning speed. $\frac{\partial y(k+1)}{\partial u(k)}$ can be approximately replaced by the function $\operatorname{sgn}\left[\frac{\partial y(k+1)}{\partial(k)}\right]$ or the differentiation $\frac{y(k+1)-y(k)}{u(k)-u(k-1)}$. In order to speed up the network learning rate, many improved BP arithmetic can be used.

\section{Stability Analysis of Self-adaptive PID Control System Based on Neural Network}

The operation of imitable human intelligent control system is asymptotically stable in a wide range. But in simulation process, the control result is usually emanated. So the theory analysis for the stability of the intelligent control is necessary. The simple analysis for the design in this paper is as shown below.

Setting Lyapunov function is:

$V(t)=\frac{1}{2} e^{2}(t)$

getting:

$$
\begin{aligned}
\Delta V(t) & =\frac{1}{2} e^{2}(t+1)-\frac{1}{2} e^{2}(t) \\
& =\frac{1}{2}[e(t+1)-e(t)][e(t+1)+e(t)] \\
& =\Delta e(t)[2 e(t)+\Delta e(t)]
\end{aligned}
$$

while $\Delta e(t) \approx\left(\frac{\partial e(t)}{\partial W}\right)^{T} \Delta W, \mathrm{~W}$ is the network weight $w_{i j}(k)(i=1,2,3 ; j=1,2)$, by the formula (6) and (7), there is $\Delta W=-\eta e(t) \frac{\partial e(t)}{\partial W}$, so $\Delta e(t)=-\eta\left\|\frac{\partial e(t)}{\partial W}\right\|^{2} e(t)$, putting it into formula (9) and there is $\Delta V(t)=-\frac{1}{2} \eta\left\|\frac{\partial e(t)}{\partial W}\right\|^{2} e^{2}(t)\left[2-\eta\left\|\frac{\partial e(t)}{\partial W}\right\|^{2}\right]$ 
By the formula (10), when learning speed satisfies $0<\eta<\frac{2}{\left\|\frac{\partial e(t)}{\partial W}\right\|^{2}}$, there is $\Delta V<0$ and the whole closed-loop system is stable.

\section{A Simulation Instance}

Setting the mathematics model of the controlled subject is $y(k)=\frac{a_{0}(k) y(k-1)+u(k-1)}{1+y^{2}(k-1)}$, the coefficient $a_{0}(k)$ is slow time-varying, $a_{0}(k)=1+0.15 \sin \left(\frac{2 k \pi}{50}\right)$. To do simulation by using neural network self-adaptive PID control system in this paper. The weight value of each network layer is $w_{i 1}(i=1,2,3)$ setting into +1 and $w_{i 2}(i=1,2,3)$ setting into -1 . Getting random data in range [-0.5, $0.5]$ for $v_{i}(i=1,2,3)$. Taking learning speed $\eta=0.3$. The input signal $r(k)$ is the square-wave with 100s cycle. The system tracking curve is as shown below.

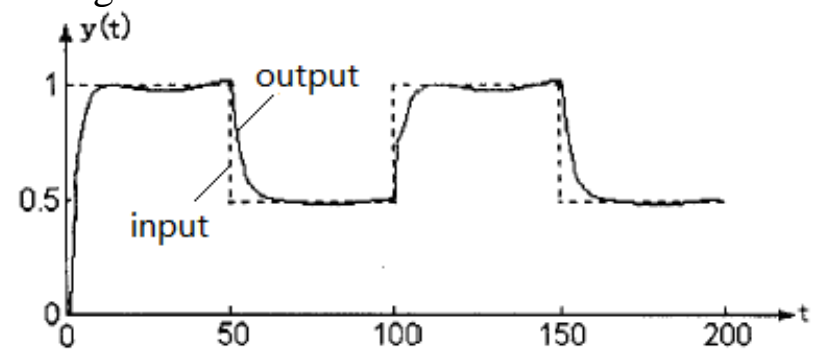

Fig. 3 The Simulation Curve of Neural Network Self-adaptive PID Control System

According to the figure above, the output of simulation object can track the system input commendably.

\section{Conclusion}

From the design method and simulation result, the neural network self-adaptive PID control system raised in this paper has the advantage of simple structure, clear parameter physical meaning, neural network self-learning and self-adaptive ability. The detail parameters and mathematics model needn't be known when control parameters are set. It is suitable for uncertain object. As the deepening of research, this type of control method will have broad prospects in control field.

\section{References}

[1] Tao Yonghua: New Type PID Controller and Application, edited by Mechanical Industry Publishing Company, China (2002), in press.

[2] Niu Yugang, Yang Chengwu: Self-adaptive Controller Base on PID Neural Network (2001)

[3] Sang-Min Kim, Woo-Yong Han. : Induction motor servo drive using robust PID-like neuro-fuzzy controller. Control Engineering Practice (2003)

[4] Han Zengjin: Self-adaptive Control, edited by Tsinghua University Publishing Company, China (1995), in press.

[5] Hu Shouren: Neural Network Application, edited byNational University of Defense Technology Publishing Company, China (1993), in press. 